\title{
Performance Evaluation of Spectrum Sensing in Cognitive Radio for Conventional Discrete-time Memoryless MIMO Fading Channel Model
}

\author{
Dipak P.Patil \\ Research Scholar, SGBAU Amravati. \\ Sandip Institute of Engineering \& Management \\ Nashik, India.
}

\author{
Vijay M.Wadhai \\ Principal, \\ Sinhgad Academy of Engineering \\ Kondhwa Pune, India.
}

\begin{abstract}
Spectrum sensing is the crucial task of a cognitive radio. Cognitive Radio (CR) have been advanced as a technology for the opportunistic use of underutilized spectrum where secondary users sense the presence of primary users and use the spectrum if it is empty, without affecting their performance. Spectrum sensing in $C R$ is challenged by a number of uncertainties, which degrade the sensing. The discrete-time memory less multiple inputs multiple output (MIMO) fading channel conventional model is implemented to appraise the performance of different spectrum sensing techniques. The signal detection in CR networks under a non parametric multisensory detection scenario is considered for performance comparison under the presence of impulsive noise. The examination focuses on performance evaluation of five different spectrum sensing mechanisms namely energy detection (ED), Generalized Likelihood Ratio Test (GLRT), Roy's largest Root Test (RLRT), Maximum Eigenvalue detection (MED) and Cyclostationary feature detection (CSFD). The analysis of the result indicates that, the sensing performance is improved in GLRT method for conventional model also it can be concluded that the performance under the conventional model can be too pessimistic in absence of impulsive noise.
\end{abstract}

Keywords-Cognitive Radio; Cyclostationary feature detection ; Energy detection; GLRT; RLRT; Spectrum Sensing.

\section{INTRODUCTION}

With the rapid growth of wireless communication, the demand for radio spectrum is expected to grow rapidly in the near future. However, radio spectrum is a limited resource and it is already very crowded. It seems that it is difficult to accommodate more wireless applications within this limited resource. On the other hand, the licensed spectrum bands are underutilized due to the current static spectrum allocation policy. This point of view is supported by recent studies of the Federal Communications Commission (FCC) [1],which reveals that in some locations or at some times of day, $70 \%$ of the allocated spectrum may be sitting idle.

Cognitive radio (CR) is originated as the paramount solution for this low usage of the available spectrum by the licensed primary users (PU). [2]. In CR the unlicensed secondary user (SU) is allowed to access the spectrum if it not utilized by the PU. However, CRs are adapted as lower priority to a PU. Sensing the available spectrum is the most vital task of the spectrum management. The basic requirement of the CR is to sense the spectrum opportunities known as spectrum holes or white spaces in the wireless environment without interfering the PU before accessing the channel [3].

Major problems for CR like multipath fading, receiver uncertainty hidden terminals and correlated shadowing observed in a non cooperative spectrum sensing can be solved by adapting the cooperative spectrum sensing techniques. This also decreases the probabilities of mis-detection and probability false alarm. The cooperative spectrum sensing is found as the most efficient method for its capability to solve hidden PU problem and also the decrease in sensing time is an added advantage to this. In [4-7], it is confirmed that the cooperative spectrum sensing is the most helpful way to fight with multipath fading and shadowing and diminish the receiver uncertainty trouble. The cooperative sensing is found handy in enhancing the sensing performance by exploiting the spatial diversity in the observations of spatially located CR users. The sensing information can be shared by the CR users and it helps to form an opinion based on the combined decisions which are more accurate than the than the individual decisions [5]. The cooperative gain is defined as the performance improvement due to spatial diversity and it can be also viewed from the viewpoint of sensing hardware. Because of the multipath fading and shadowing, the measured signal-to-noise ratio (SNR) of the received primary signal can be enormously small thus the detection of becomes a complex task. The ability to detect the week signal defines the receiver sensitivity. The sensitivity necessities will be forced on a severe implementation complexity and the associated hardware cost. Increasing the sensitivity is not the solution for improving the detection performance when the SNR of the PU signals is below a definite threshold level known as a SNR wall [8].

Fig.1 illustrates that the cooperative sensing scheme is useful to improve the sensing performance degraded because of multipath fading and shadowing, without increasing the implementation cost of CR devices by setting the sensitivity to the same level of nominal path loss [6]. 


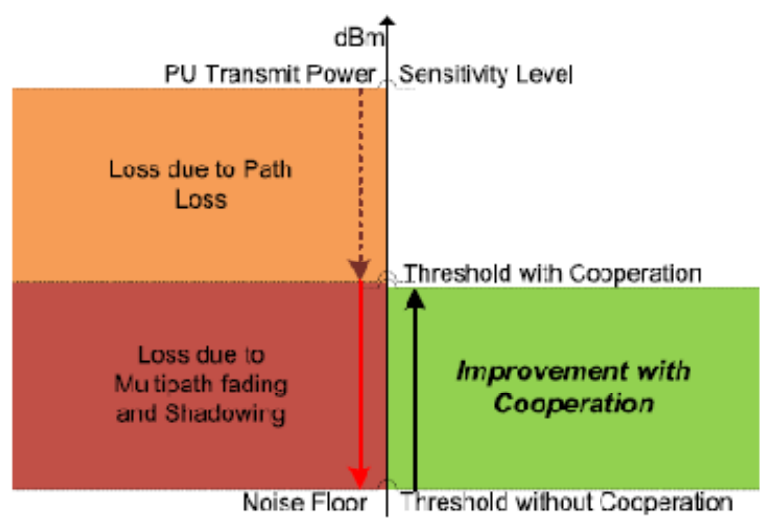

Figure 1. Sensitvity Improvement with cooperative sensing [6]

Centralized or distributed cooperation are the two different ways of implementing the cooperative sensing network, which can be build by cooperation among CRs.

As shown in Fig.2, the central unit coordinates for collecting the sensing information from cognitive devices in the centralized sensing. It directly controls the CR traffic by recognizing the vacant spectrum and broadcasts this information to other CRs.

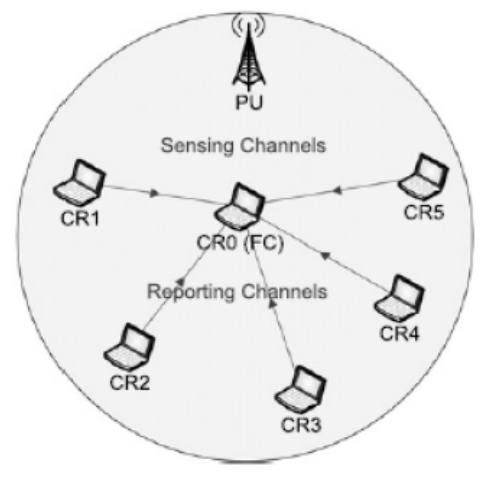

Figure 2. Centralized cooperative sensing

At the access point all the sensing results are collected, which is the central position [9]. The objective is to improve the fading effects of the channel and boost detection performance. Resulting detection and false alarm rates are given in [10] for the sensing algorithm used in [9]. Data fusion is the process of combining the reported sensing results for making the cooperative decision, where user sends quantized edition of their local decisions to central unit fusion center (FC) [11]. Then the FC decides the occupancy of the channel. In order to satisfy the bandwidth constraints arrived due to large number of users the local observations of cognitive radios are quantized to one bit (hard decisions) which reduces the bandwidth [12]. In addition, only the CRs with reliable information are permitted to report their decisions to the central unit.

The basic spectrum sensing techniques are matched filter detection MFD [13-16], ED [17-20] and CSFD [21-23].Each technique is unique in itself and possessing certain advantages and drawbacks. In CSFD, it is necessary to understand thecyclic frequencies of the primary signal, which practically may not be available to the secondary users. The high computational complexity requirement is another disadvantage of CSFD.The (MFD) is assumed to be an optimal signaldetection method. But it is necessary to have the prior knowledge of the primary user, e.g., modulation type, pulse shaping, and synchronization of timing and carrier.And in MFD, for each PU, the CR will require a committed receiver and this requirement is makes it difficult for practical implementation [24]. From [25- 28] it is observed that the eigenvalue-based spectrum sensing techniques is found as the best amongst existing sensing methods. The eigenvalue-based spectrum sensing methods ahs overcome the limitations of the previously discussed methods. The prior knowledge of the transmitted signal is not essential in this method. Also the most basic sensing method the ED is reasonably sensitive to the accuracy of the expected noise variance [29]. We considered the effect of impulsive noise, which consists of repetitive or non-repetitive pulses with a random intensity duration and occurrence for investigating the performance of the eigenvalue-based spectrum sensing scheme in perspective of spectrum sensing [30-31].

The rest of the paper is organized as follows. Section II briefly describes the system model for all spectrum detection techniques under test. Simulation setup for performance analysis of the five spectrum sensing techniqueswith configuration of parameters is presented in Section III .Simulation results with comparative sensing performance is illustrated in Section IV. Finally conclusions are drawn in Section V.

\section{SYSTEM MODEL}

Memory-less linear discrete-time multiple input multiple output (MIMO) fading channel is used for modeling the received samples for single-receiver, multi-sensor and multiple-receiver, single-sensor cognitive devices in the data fusion cooperative spectrum sensing. It is also known as conventional model (C-model).It is the static model in which channel remains idle, we consider Additive White Gaussian Noise (AWGN) channel. Because of limitation of no signal processing performed by the C-model, it cannot be used for multiple CR receivers, as the samples collected by each CR are considered and forwarded to the fusion center directly. Hence modifications are essential in the C-model.

The binary hypotheses are applied to the system model with the choices are labeled as $H_{0}$ and $H_{1}$, which represent sensed states for presence and absence of the signal [32-33]. The system model of detection is under the test of following two hypotheses $H_{0}$ and $H_{1}$ [33].

$H_{0}$ : represents the absence of the signal and presence of only noise.

$H_{1}$ :represents the presence of both signal and noise.

Thus, for the two state hypothesis mathematically one can obtain following three cases : 
1) Probabilty of Detection $\left(P_{d}\right)$ : i.e $P\left(H_{l} / H_{l}\right)$, correpspons $H_{l}$ to be true for the presence of primary signal.

2) Probabilty of Missed Detection $\left(P_{m d}\right)$ : i.e $P\left(H_{0} / H_{1}\right)$ ,correpspons $H_{0}$ to be true for the presence of primary signal.

3) Probabilty of False Alarm $\left(P_{f a}\right)$ : i.e $P\left(H_{1} / H_{0}\right)$,correpspons $H_{l}$ to be true for the presence of primary signal.

Probability of detection $\left(P_{d}\right)$ and probability of false alarm $\left(P_{f a}\right.$ can be evaluated respectively as [20],

$$
\begin{gathered}
P_{d}=P\left(Y^{\prime}<\gamma \mid H_{1}\right) \\
P_{f}=P\left(Y^{\prime}<\gamma \mid H_{0}\right)
\end{gathered}
$$

The plot of $P_{d}$ versus $P_{f a}$ is known as the receiver the operating characteristic (ROC) curves as they vary with the decision threshold $\gamma$. Here, $P$ represents the probability of a given event, $T$ is the detection-dependent test statistic and $\gamma$ is the decision threshold. The value of $\gamma$ is decided on the requirements for the spectrum sensing performance, which is typically evaluated through ROC.

The multi-sensor detection setting is considered, where the detectors test statistic is constructed from $K$ sensors (receivers or antennas) and $N$ time samples. Let $y(n)=\left[y_{1}(n) \ldots . y_{k}(n)\right]^{T} \mathrm{e}$ the $K \times 1$ received vector at time $n$, where the element $y_{k}(n)$ is the discrete baseband complex sample at receiver. The received power under $H_{0}$, vector consists of $K$ complex Gaussian noise samples with zero mean and variance $\sigma_{v}^{2}$.

$$
y(n) \mid H_{0}=v(n)
$$

Where, $v(n) \sim N C\left(0_{K \times 1}, \sigma_{v}^{2}, I_{K \times K}\right)$.Under $H_{l}$, in compare, the received vector contains signal plus noise,

$$
y(n) \mid H_{1}=x(n)+v(n)=h s(n)+v(n)
$$

Where, $s(n)$ is the transmitted signal sample, modeled as a Gaussian $^{2}$ random variable with zero mean and variance $\sigma_{\mathrm{s}}^{2}$ and $h$ is the $K \times 1$ unknown complex channel vector. The channel is assumed to be memory less and constant during the detection time. Under $H_{1}, \mathrm{SNR}$ at the receiver is defined as,

$$
\rho=\frac{E\|x(n)\|^{2}}{E\|v(n)\|^{2}}=\frac{\sigma_{\mathrm{s}}^{2}\|h\|^{2}}{\sigma_{v}^{2} K}
$$

Where, $\|$.$\| denotes Euclidean (L2) norm.$

The received samples are stored by the detector in the $K \times N$ matrix,

$$
y \cong[y(1) \ldots . . y(N)]=h(s)+v
$$

Where, $s \cong[s(1) \ldots . . s(N)]$ is a $1 \times N$ signal vector and $v \cong[v(1) \ldots . . v(N)]$ is a $K \times N$ noise matrix. The sample covariance matrix $R$ is then defined as,

$$
R \cong \frac{1}{N} Y Y^{H}
$$

Let $\lambda_{1} \geq \ldots . \geq \lambda_{K}$ be the eigenvalues of $R$ (without loss of generality, sorted in decreasing order).

The test figures for GLRT, CSFD, MED, ED and RLRT are correspondingly considered according to [27-28].

$$
\begin{gathered}
T_{G L R T}=\frac{\lambda_{1}}{\frac{1}{m} \operatorname{tr}(R)}=\frac{\lambda_{1}}{\frac{1}{m} \sum_{i=1}^{m} \lambda_{i}} \\
T_{C S F D}=\frac{\lambda_{1}}{\lambda_{i}} \\
T_{M E D}=\frac{\lambda_{1}}{\sigma^{2}} \\
T_{E D}=\frac{\|Y\|_{F}^{2}}{m n \sigma^{2}}=\frac{1}{m \sigma^{2}} \sum_{i=1}^{m} \lambda_{i}, \\
T_{R L R T}=\frac{\lambda_{1}}{\sigma_{v}^{2}}
\end{gathered}
$$

Where, $\sigma^{2}$ is the thermal noise power, understood to be known and with identical value in each sensor input, and $\operatorname{tr}()$ and \|\|$_{F}$ are the trace and the Frobenius norm of the underlying matrix respectively.

\section{Simulation SETUP}

For C-model the parameters configured for simulation are as,

$m$ : Antennas in $\mathrm{CR}$

$n$ : Number of received samples collected from primary transmitter.

$N_{e}$ : Number of Monte Carlo events simulated.

The sensing techniques under test are ED, MED, CSFD, GLRT and RLRT.

Type of transmitted signal (noise, BPSK, QAM or user defined module).

Two different simulation processes are carried out. For the first simulation type, the SNR is kept at fixed value in $\mathrm{dB}$ and the decision threshold range is varied between minimum and maximum values. And second process of simulation is carried out by defining three parameters like a preset threshold, and the minimum and the maximum SNR values in $\mathrm{dB}$ with the number of points within the SNR range.

\section{Simulation Results}

Probability of Detection $\left(P_{d}\right)$ and Probability of False alarm $\left(P_{f a}\right)$ are the performance measurement metrics considered for analysis. The performance of different spectrum sensing techniques is illustrated by the receiver 
operating characteristics (ROC), curve which is a plot of $P_{d}$ versus $P_{f a \text {. }}$

The performance measurements parameters set for simulation scenario one as, $m=8, n=50, S N R=-10 d B$, no. of Monte Carlo events simulated $=1000$ and minimum to maximum threshold levels set in the range $\gamma=0.78$ to 1.1 with 8 threshold events. For the second simulation setup the threshold level is kept fixed at value $\gamma=1.4$ and SNR is varied in between the range of $-10 \mathrm{~dB}$ to $20 \mathrm{~dB}$.

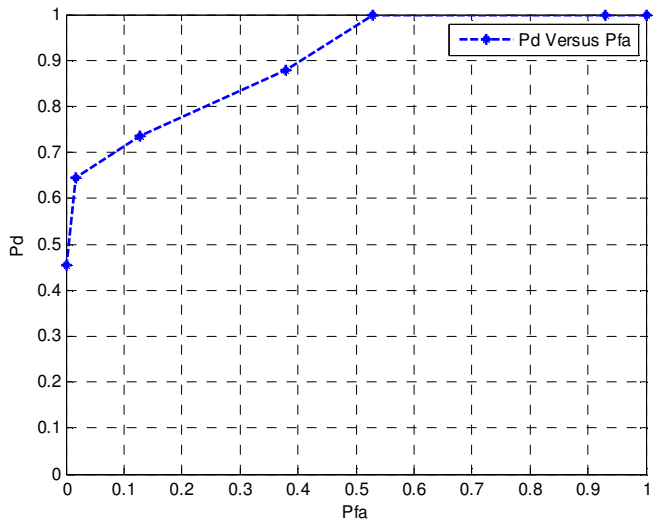

Figure 3. ROC Curve when $S N R=-10 \mathrm{~dB}$ for $m=8$ and $n=50$

Fig.3, represents the ROC curve for ED method, it is evident from the graph that the value of $P_{d}$ is comparatively high for the less $P_{f a}$ values. But, as $P_{f a}$ values are increased, $P_{d}$ values are also increased drastically. Thus, the detection is improved in energy detector at low SNR values. We reiterate that this is valid for all detection techniques considered here, though results were presented only for the ED.

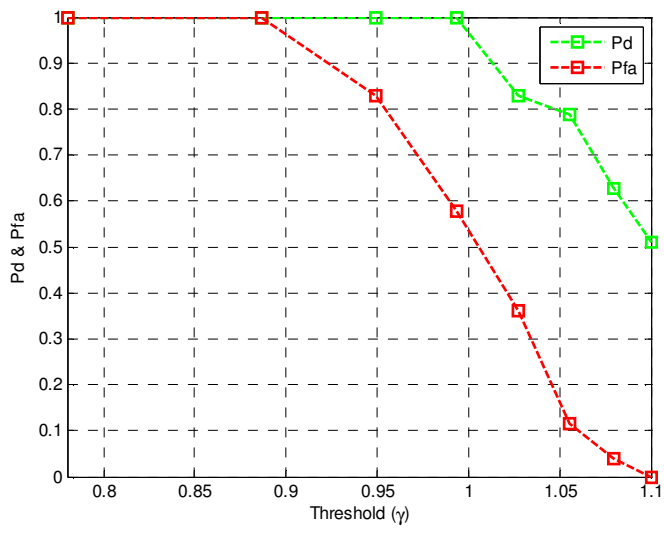

Figure 4. $P_{d} P_{f a}$ Vs Threhold

Fig.4. shows comparison of $P_{d} P_{f a}$, with respect to the change in threshold levels from $\gamma=0.78$ to 1.1. Fixed a given threshold, the behavior of $P_{f a}$ is shown, it is evident from the results that $P_{d}$ is improved and these curves are useful for computing the threshold necessary to achieve a given false alarm rate.

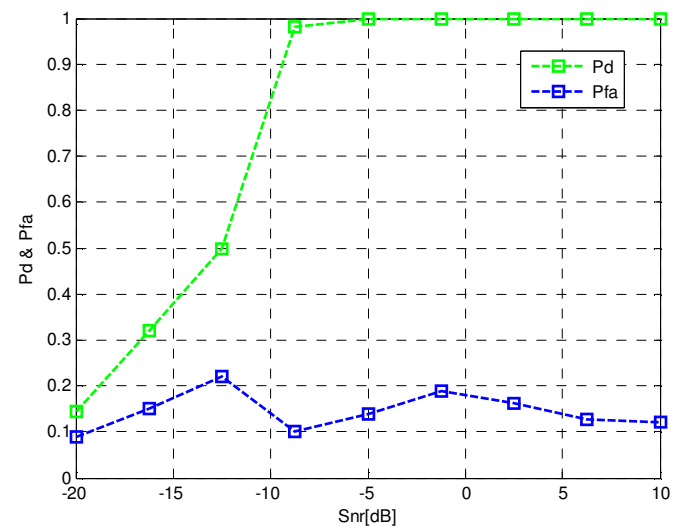

Figure 5. $P_{d} P_{f a}$ Vs $S N R$

Fig.5, presents the relation between the $P_{d}$ and $P_{f a}$, with respect to the SNR. It is inveterate that with respect to the increase in SNR the value of $P_{d}$ is also increased. As the value of $P_{f a}$, varies there is significant improvement in $P_{d}$ is obtained.

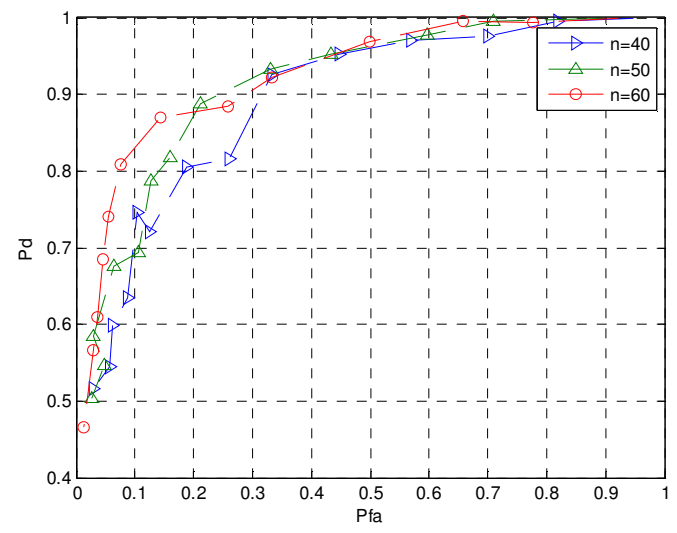

Figure 6. ROC Curve for ED Method with $m=8, S N R=-10 \mathrm{~dB}$

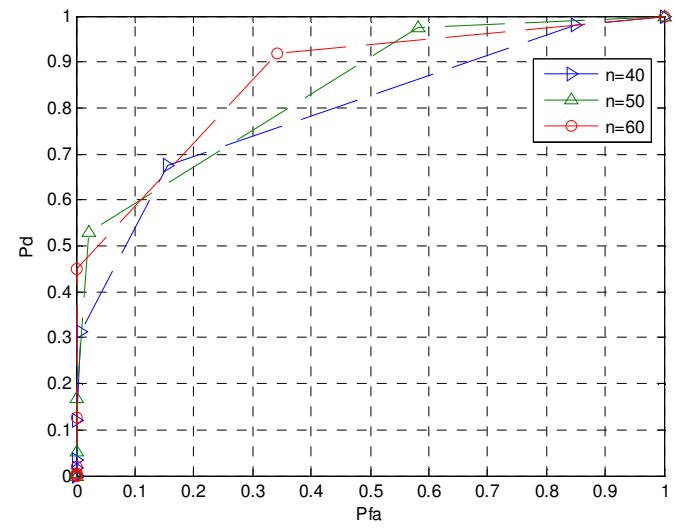

Figure 7. ROC Curve for GLRT method with $m=8, \mathrm{SNR}=-10 \mathrm{~dB}$ 


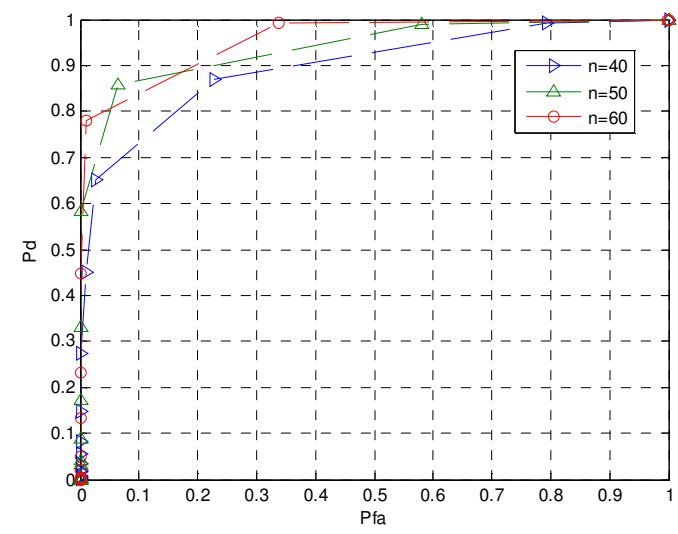

Figure 8. ROC Curve for CSFD Method with $m=8, S N R=-10 \mathrm{~dB}$

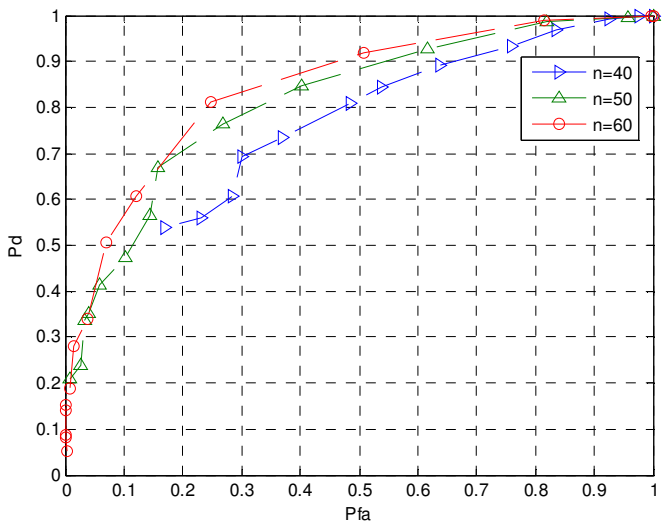

Figure 9. ROC Curve for MED Method with $m=8, S N R=-10 \mathrm{~dB}$

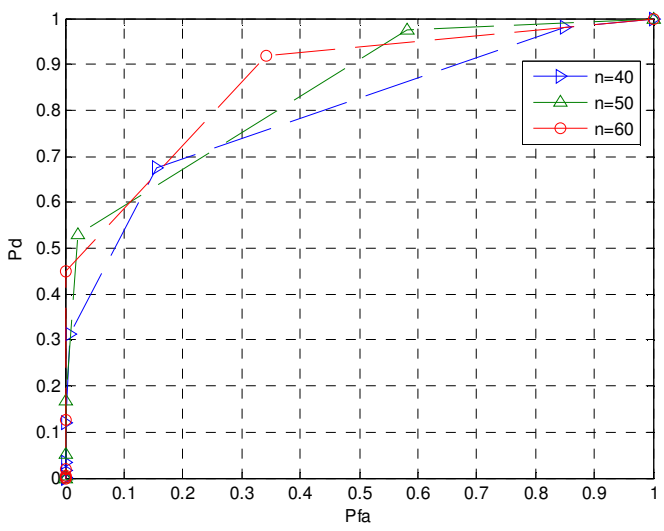

Figure 10. ROC Curve for RLRT Method with $m=8, S N R=-10 \mathrm{~dB}$

Fig. 6-10 shows ROC curve for different values of the number of collected samples $(n)$ concerning the Probability of Detection $P_{d}$ and Probability of False alarm $P_{f a}$.With reference to the $(n)$, we set different minimum to maximum threshold levels as follows. For $n=40$ the threshold levels set are $\gamma_{\min }=2.8$ and $\gamma_{\max }=6.5$ and for $n=50, \gamma_{\min }=2.8$ and $\gamma_{\max }=6.5$ and for $n=60, \gamma_{\min }=2$ and $\gamma_{\max }=4.5$. From the obtained results, it is confirm that with the greater threshold value, the values of $P_{d}$ and $P_{f a}$ are smaller. Similarly, for smaller threshold, $P_{d}$ and $P_{f a}$ tend to 1 . Increase in sensing performance is observed with increase in $(n)$.

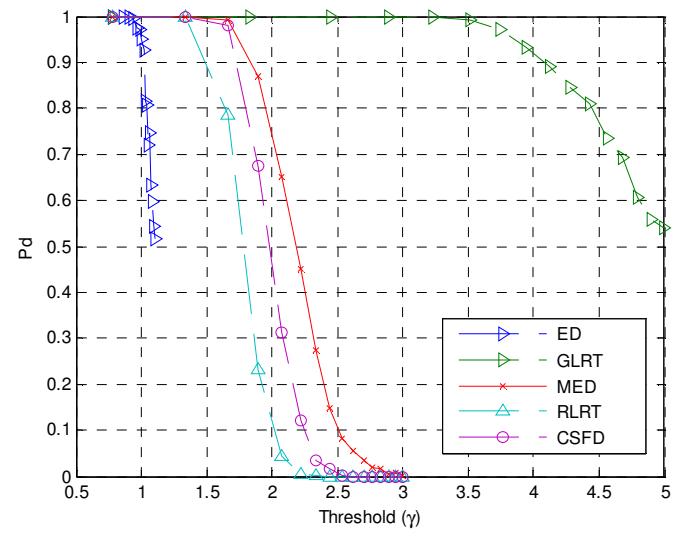

Figure 11. $P_{d}$ Vs Threshld for all sensing methods

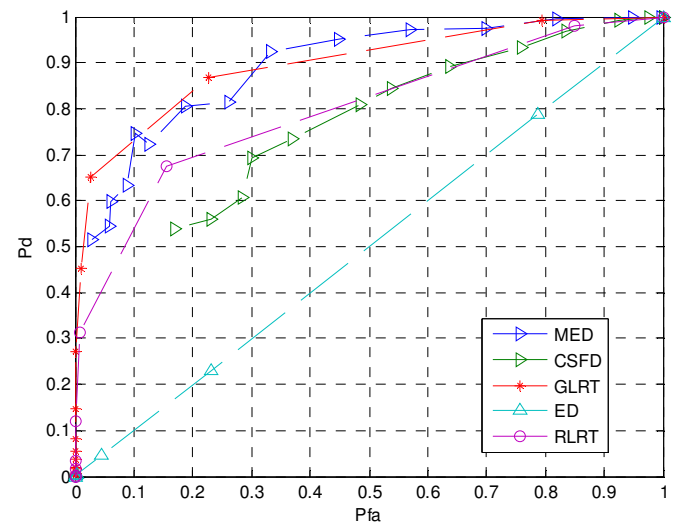

Figure 12. ROC Curve for all detection techniques

Fig. 11-12, illustrates the performance comparison of all the detection methods under test namely, GLRT, CSFD, MED, ED and RLRT. From the comparative plot, it is evident that the MED and GLRT methods provide better sensing performance than the other detection methods for variable threshold level with fixed SNR value. ED method shows poor performance with $P_{d} P_{f a}$. The analysis of the results indicates that, the sensing performance is improved in GLRT by the improved probability of detection as compared to other methods for conventional model.

\section{CONCLUSIONS}

In this paper, the performance of different detection methods is evaluated with respect to the number of collected samples without any kind of signal processing on them with multiple CR receivers. The received samples are modeled for single-receiver, multi-sensor and multiple-receiver, singlesensor cognitive devices in the data fusion cooperative spectrum sensing. The memory-less linear discrete-time 
multiple input multiple output (MIMO) fading channel is used. Closed form expressions for probability of detection and false alarm over different sensing methods are evaluated. Simulation results verify that, the GLRT detection method produces better sensing performance than the other detection methods, particularly in the case of limited samples in conventional model even if no signal processing is involved between the cooperating CRs.

In contrast to earlier work, the conventional model can be useful for performance comparison of sensing techniques and provide reasonably better results. Moreover the comparison of results helps for performance evaluation and of sensing methods in Cognitive Radio networks. It is believed that the work carried out in this paper is useful to understand the performance in Cognitive Radio network for selection of best sensing method for discrete-time memory less MIMO fading channel. In future work attention can be given on the computation of closed form Analysis by using more realistic approach with typical CR signal processing tasks.

\section{REFERENCES}

[1] Federal Communications Commission,"Spectrum policy task force report," ET Docket No. 02-135; November 2002.

[2] J.Mitola , Maguire G.Q., “Cognitive Radio: making software radios more personal," IEEE Personal Commu.Mag., 1999;6:13-8.

[3] J. Mitola, III, "Cognitive radio for flexible mobile multimedia communications," in Proc. IEEE Mobile Multimedia Commun. Conf., San Diego, CA, Nov. 1999, pp. 3-10.

[4] D. Cabric, R. Brodersen ,S. Mishra, "Implementation issues in spectrum sensing for cognitive radios," in: Proc. of Asilomar Conf. on Signals, Systems, and Computers, vol. 1, 2004, pp. 772-776.

[5] A. Ghasemi, E. Sousa, "Collaborative spectrum sensing for opportunistic access in fading environments," in: Proc. of IEEE DySPAN 2005, 2005, pp. 131-136.

[6] A.Sahai,S. Mishra, R. Brodersen, "Cooperative sensing among cognitive radios", in: Proc. of IEEE ICC 2006, vol. 4, 2006, pp. 1658-1663.

[7] A. Sahai,R. Tandra, "SNR walls for signal detection," IEEE Journal of Selected Topics in Signal Processing 2 (1) (2008) 4-17.

[8] I.F.Akyildiz.,"NeXt generation/dynamic spectrum access/cognitive radio wireless networks: a survey," In: Elsevier computer networks 2006,vol.50;2006.p.2127-59.

[9] F. Jondral,J. Hillenbrand and T. Weiss, "A diversity approach for the detection of idle spectral resources in spectrum pooling systems," in Proc. of the 48th Int. Scientific Colloquium, Ilmenau, Germany, Sept. 2003, pp. 37-38.

[10] F. Jondral,J. Hillenbrand and T. Weiss, "Calculation of detection and false alarm probabilities in spectrum pooling systems," IEEE Commun. Lett., vol. 9, no. 4, pp. 349-351, Apr. 2005.

[11] A. Huttunen, H. V. Poor, J. Lunden, and V. Koivunen, "Spectrum sensing in cognitive radios based on multiple cyclic frequencies," in Proc. IEEE Int. Conf. Cognitive Radio Oriented Wireless Networks and Commun. (Crowncom), Orlando, Florida, USA, July/Aug. 2007.

[12] C. Sun, K. B. Letaief, and W. Zhang, "Cooperative spectrum sensing for cognitive radios under bandwidth constraints," in Proc. IEEE Wireless Commun. and Networking Conf., Hong Kong, Mar. 2007, pp. 1-5.

[13] A. Sahai and D. Cabric, "Spectrum sensing: Fundamental limits and practical challenges," In proc. IEEE Int.Symp.New Fronties DySPAN (Tutorial),Baltimore,MD Nov.2005.

[14] S. M. Kay, "Fundamentals of Statistical Signal Processing: Detection Theory," Upper Saddle River, NJ: Prentice-Hall, 1998.

[15] D. G. Daut ,H. S. Chen and W. Gao, "Signature based spectrum sensing algorithms for IEEE 802.22 WRAN," in Proc. IEEE Int. Conf. Commun., Glasgow, U.K., Jun. 24-28, 2007, pp. 6487-6492.
[16] A. Tkachenko, D. Cabric, and R. W. Brodersen, "Spectrum sensing measurements of pilot, energy, and collaborative detection," in Proc. MILCOM, Washington, DC, Oct. 23-25, 2006, pp. 1-7.

[17] H Urkowitz, "ED of unknown deterministic signals," Proc. IEEE, vol. 55, pp. 523-531, Apr. 1967.

[18] C.Tellambura, H. Jiang and S.Atapattu, "Analysis of Area under ROC curve of energy detection" $\mathbb{E E E}$ Transactions On Wireless Communications, Vol. 9, No.3, pp. 1216-1225, 2010

[19] V. I. Kostylev, "Energy detection of a signal with random amplitude," in Proc. IEEE ICC, Apr. 2002, vol. 3, pp. 1606-1610.

[20] F.F. Digham, M.S. Alouini and M.K. Simon, "Energy detection of unknown signals over fading channels", IEEE Transactions on Communications, Vol. 5, No.1, pp. 21- 24, 2007.

[21] W. A. Gardner, "Signal interception: A unifying theoretical framework for feature detection," IEEE Transactions on Communications, vol. 36 , no. 8, pp. 897-906,Aug. 1988.

[22] W. Gardner, "A new method of channel identification," IEEE Transactions on Communications, vol. 39 , no. 6, pp. 813-817, Jun 1991.

[23] K. E. Nolan,L. E. Doyle and P. D. Sutton, "Cyclostationary signatures in practical cognitive radio applications," IEEE J. Sel. Areas Commun. vol. 26, no. 1, pp. 13-24, Jan. 2008

[24] D. Cabric, S. M. Mishra, and R. W. Brodersen, "Implementation issues in spectrum sensing for cognitive radios," in Proc. 38th Asilomar Conf. Syst. Comput., Pacific Grove, CA, Nov. 7-11, 2004, pp. 772-776.

[25] Y.Liang, Y.Zeng, " Eigen value-based spectrum sensing algorithms for cognitive radio.Communications," IEEE Transactions on communications, 2009;57(6):1784-93. 2005.

[26] Caijun Zhong, Kortun A, Papadias CB, Sellathurai M, and Ratnarajah T "On the Performance of Eigenvalue-Based Cooperative Spectrum Sensing for Cognitive Radio," IEEE Journal of Selected Topics in Signal Processing,. 2011;5(1):49-55.

[27] B Nadler, F.Penna and R.Garello ., "Performance of Eigenvalue-Based Signal Detectors with Known and Unknown Noise Level ," In procedddings of IEEE International Conference on communications,ICC,Kyto,Japan,5-9 June 2011;pp.1-5.

[28] A.A. dos Anjos,D.A.Guimarães and R.A.de Souza,"Simulation Platform for Performance Analysis of Cooperative Eigenvalue Spectrum Sensing with a Realistic Receiver Model Under Impulsive Noise ," Vehicular Technologies-Deployment and Applications, 2013.

[29] A. Sahai and R. Tandra, "Fundamental limits on detection in low SNR under noise uncertainty," in Proc. Int. Conf. Wireless Netw., Commun.Mobile Comput., Maui, HI, Jun. 13-16, 2005, pp. 464-469.

[30] Calvo-Ramon, L.M.; Sanchez, M.G., Martinez-Rodriguez-Osorio, R.; de Haro-Ariet, L.,"Performance evaluation of W-CDMA in actual impulsive noise scenarios using adaptive antennas",IEEE Proc. Commun. 2004, 151, 589-594.

[31] M.Budsabathon, S.Hara, "Robustness of OFDM Signal Against Temporally Localized Impulsive Noise", In Proceedings of the IEEE VTS 54th Vehicular Technology Conference, VTC 2001 Fall, Atlantic City, NJ, USA, 7-11 October 2001; Volume 3, pp. 1672-1676.

[32] M.Srinath,P.Rajasekaran and R.Vishwanathan, " Introduction to stastaticall signal Processing and Applications," Prientice Hall,Eaglewood Cliffs, 07692,1996.

[33] D. Goodmanand,Yates R.D, "Probability and Stochastic Processes: A Friendly Introduction for Electrical and Computer Engineers.," 2nd ed. 2004.

[34] C. Cordeiro,K. Challapali and S.Shankar, "Spectrum agile radios: utilization and sensing architectures,"in Proc. IEEE Int .on New Frontiers in Dynamic Spectrum Access Networks, Baltimore Maryland, USA, Nov. 2005, pp. 160-169. 